\title{
A Practical Method for Grid Structures Damage Location
}

\author{
Zhefu Yu' ${ }^{1}$ and Linsheng Huo ${ }^{2}$ \\ ${ }^{1}$ Transportation Equipment and Marine Engineering College, Dalian Maritime University, Dalian, Liaoning 116026, China \\ ${ }^{2}$ Dalian University of Technology, Linggong Road No. 2, Integrated Building 4, 219-B, Dalian, Liaoning 116024, China \\ Correspondence should be addressed to Zhefu Yu; yuzf629@sina.com
}

Received 29 September 2014; Revised 29 January 2015; Accepted 9 February 2015

Academic Editor: Christos Riziotis

Copyright (C) 2015 Z. Yu and L. Huo. This is an open access article distributed under the Creative Commons Attribution License, which permits unrestricted use, distribution, and reproduction in any medium, provided the original work is properly cited.

\begin{abstract}
A damage location method based on cross correlation function, wavelet packet decomposition, and support vector machine was proposed for grid structure. The approximate damage positions in grid structures could be determined through the peak abrupt changes of the cross correlation function that was produced by two vibration responses of adjacent measuring points. The vibration response was decomposed into eight bands by wavelet packet in order to accurately locate damage rods. The energy distribution in eight bands was used as a feature vector. SVM is trained to locate damaged bar elements in grid structures. Numerical analysis results showed that this method had good accuracy.
\end{abstract}

\section{Introduction}

Grid structures suffer from all kinds of damage during service, due to environmental effects, natural disasters, and human factors. For the maintenance of grid structures, accurately locating damage position is very critical.

The traditional methods of damage location are based in displacement measurement and strain measurement, but they are damaging to the structures [1]. According to the theory of structural dynamics, structural damage can affect the dynamic characteristics of structures. Therefore, damage location methods based on vibration analysis have attracted much attention in the past twenty years [2]. The modes of structural damage could be identified by analyzing modal parameters. The modal parameters include natural frequencies [3], vibration modes $[4,5]$, and other parameters $[6,7]$. For a complex structure, the high natural frequencies are difficult to be measured. Precise measurement of vibration modes requires more measuring sensors [8]. The modal parameters are affected easily by signal noise, structure characteristics, and human factors. The damage location methods based on modal parameters cannot reach the expected result.

Despite the fact that the damage detection methods integrated signal processing [9], pattern recognition and artificial intelligence $[10,11]$ are the developing direction in recent years; such methods were only applied to some of simple structures. Because the number of bar element in grid structure is huge, and the number of damage mode is enormous, to get the damage location of grid structure, a larger number of damage mode samples are required to train classifier, which will bring huge workload for building samples with finite element methods.

This paper proposed a damage location method which integrated the cross correlation function of the random vibration, wavelet packet decomposition, and SVM. The proposed method includes two steps. The first step is the approximate damage location. The damaged basic units were found through the peak abrupt changes of the cross correlation function [12]. In the second step, wavelet packet and support vector machines are used in determining the damaged bars in the basic units.

\section{Cross Correlation Function, Wavelet Packet Decomposition, and SVM}

2.1. The Conception of Cross Correlation Function. Cross correlation function can reflect the correlation between two random vibration signals. The correlation changes with the time interval of the two signals. If a structure is subjected to 
a random excitation, the response of two adjacent measuring points can be regarded as two stationary random processes $x_{1}$ and $x_{2}$. The cross correlation function is shown as

$$
\begin{aligned}
\varphi_{x_{1} x_{2}}(\tau) & =E\left[x_{1}(t) x_{2}(t+\tau)\right] \\
& =\iint x_{1} x_{2} p\left(x_{1} x_{2}\right) d x_{1} d x_{2}
\end{aligned}
$$

where $\phi_{x_{1} x_{2}}$ is the cross correlation function; $\tau$ is the time interval; $E[\cdot]$ means the expecting value; $p\left(x_{1} x_{2}\right)$ denotes joint probability distribution function.

If the random vibration responses are ergodic, the cross correlation function can be derived through the time-history of one random process. It is shown as

$$
\varphi_{x_{1} x_{2}}(\tau)=\lim \lim _{T \rightarrow \infty} \frac{1}{T} \int_{0}^{T} x_{1}(t) x_{2}(t+\tau) d t .
$$

In the numerical analysis, the vibration response in each measuring point is a discrete time series. The integral of cross correlation function can not be acquired by (2). It can be replaced by the summation formula as shown in

$$
\varphi_{x_{1} x_{2}}(k)=\frac{1}{N} \sum_{i=1}^{N-k} x_{1}(i) x_{2}(i+k), \quad(k=0,1, \ldots, N),
$$

where $N$ is the number of sampling points.

In a cross correlation function, the largest amplitude as shown in (4) is defined as peak in this paper:

$$
r_{l, l+1}=\left|\varphi_{x_{l} x_{l+1}}(\tau)\right|_{\max }=\left|\varphi_{x_{l} x_{l+1}}(k)\right|_{\max } .
$$

According to the characteristics of grid structures, measuring points are arranged on the bottom nodes uniformly. Cross correlation functions can be acquired by adjacent measuring points. Every cross correlation function has a peak. Thence, a peak matrix for the entire grid structure can be derived [12]. By introducing the peak matrix, the influence of noise pollution in measurement signals can be reduced $[13,14]$. In case of one damage mode of a grid structure, the peak matrixes obtained from same spectrum vibrations are highly similar. After normalizing treatment, they are almost identical. Different damage modes produce different peak matrixes. By comparing the peak matrix of the damaged structure with the peak matrix of the intact structure, the approximate damage position would be determined.

2.2. Wavelet Packet Decomposition. Wavelet packet decomposition is derived from the wavelet analysis, which is a tool for multilevel band analysis and signal reconstruction. Wavelet packet decomposition can decompose the highfrequency portion of a signal more narrowly than the wavelet analysis. The different bands of a signal have different energy. The energy distribution of the vibration responses in grid structure may reflect the damage position.

2.3. SVM. SVM proposed by Vapnik is a machine learning algorithm based on statistical learning theory [15]. It minimizes actual risk through seeking minimal structural risk.

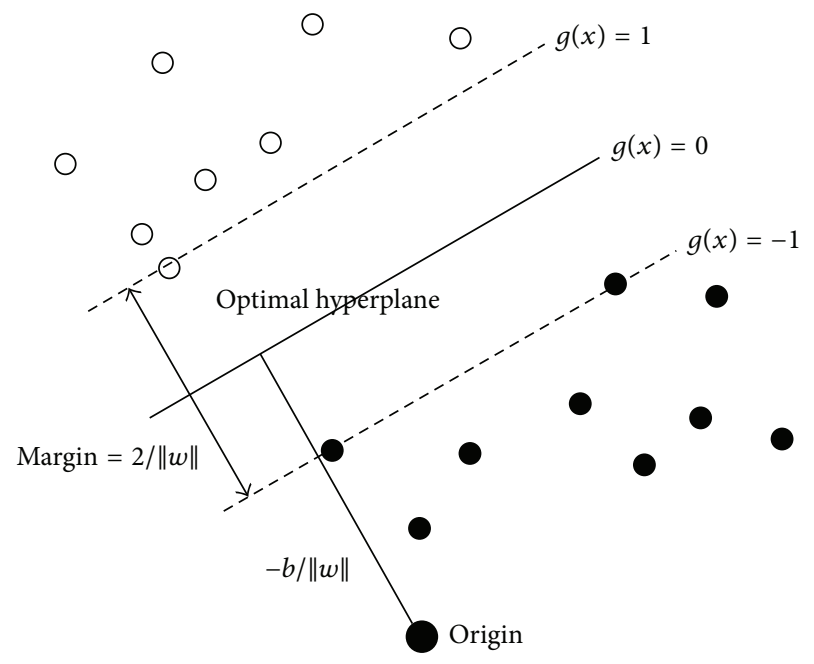

Figure 1: Support vector machine.

It can get a good learning result in the case of small sample size. Since the SVM algorithm is a quadratic optimization problem, the resulting solution is globally optimal.

The explanation of SVM starts with a set of training data $\left(x_{1}, y_{1}\right), \ldots,\left(x_{l}, y_{l}\right)$, where $x_{i} \in R^{n}$ is an $n$-dimension vector and $y_{i} \in\{-1,+1\}$ is the class label of the $i$ th sample. The optimal hyperplane $(w \cdot x)+b=0$ divides the training data into two classes. The basic idea of SVM is to maximize the margin between the positive samples and the negative samples. Figure 1 shows that the training examples can be linearly separated into two classes. In general, it is not necessary to separate training examples into each class without error. The variable $\xi_{i} \geq 0$ is introduced for misclassification errors; $C>0$ is a constant. Then, this optimization problem is defined as follows:

$$
\begin{aligned}
& \min _{w} \phi(w)=\|w\|^{2}+C \sum_{1}^{l} \xi_{i} \\
& \text { s.t.: } y_{i}\left(w \cdot x_{i}+b\right) \geq 1, \quad i=1, \ldots, l
\end{aligned}
$$

In (5), the first term $\|w\|^{2}$ specifies the size of the margin, and the second term $C \sum_{1}^{l} \xi_{i}$ represents the cost of the misclassification. The decision function $f(x)$ can be written as

$$
f(x)=\operatorname{sgn}\left(\sum_{i} \alpha_{i} y_{i}\left(x_{i} \cdot x\right)+b\right),
$$

where $\alpha_{i} \geq 0$ are Lagrange multipliers. $\alpha_{i}$ corresponds to the $i$ th sample. When the maximal margin hyperplane is found in feature space, the $\alpha_{i}$ corresponding to the points close to the hyperplane are greater than zero, and these points are called the support vectors. The $\alpha_{i}$ corresponding to other points are equal to zero, which means that the representation of the hyperplane is solely given by the support vectors. 


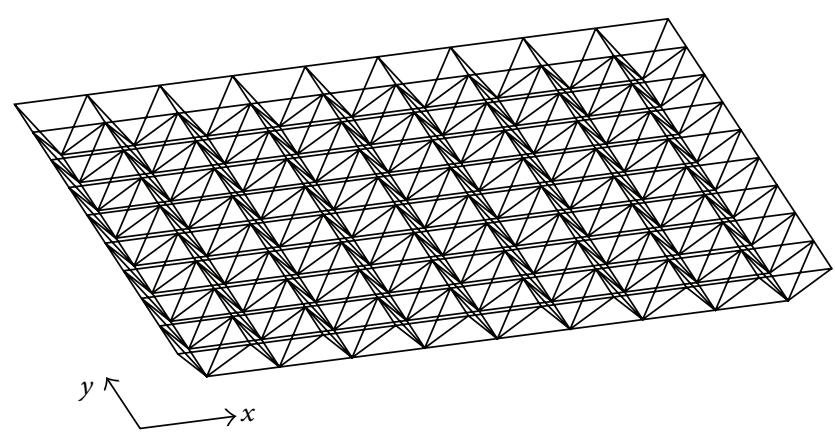

FIGURE 2: The grid structure.

The kernel function is defined as $\Phi(u) \otimes \Phi(v)=K(u, v)$ for a non-linear SVM classifier. After using a kernel function, (7) can be rewritten as

$$
f(x)=\operatorname{sgn}\left(\sum_{i} \alpha_{i} y_{i} K\left(x_{i}, x\right)+b\right) .
$$

Classical SVM algorithm is only a two-class classifier, but general problems need multiclass classifiers. It was solved by combining multiple two-class classifiers. There are many combination methods, such as one to all, one to one, and decision tree. In this paper, the combination method of one to one is used to realize the multiclass classification.

\section{Damage Location of Grid Structure}

3.1. Grid Structure. A double square pyramid grid was chosen to verify the damage location method proposed in this paper. The grid is square with the side length of $30 \mathrm{~m}$ and height of $1.5 \mathrm{~m}$. The bars are seamless steel pipe with the crosssectional dimension of $40 \mathrm{~mm}$. In the case of support, there is fixed hinge bracket in the four corners of the grid, which is shown in Figure 2. The SAP2000 software was applied for FEM analysis.

Excitation is an important part of the dynamic analysis. It is directly related to the analysis accuracy. Excitation can be divided into artificial excitation and environmental pulsation. Because the damage location indicator proposed in this paper is the peak of cross correlation function, the noise control in the acceleration response signal of measuring point is unimportant $[6,7]$. So this method uses environmental pulsation as the signal source. In the process of finite element calculation, an acceleration signal source is a white noise signal generated by MATLAB. The acceleration amplitude delivered to the grid network through fixed hinge bracket is $2 \times 10^{-3} \mathrm{~m} / \mathrm{s}^{2}$. The sampling interval is $0.01 \mathrm{~s}$, and the sampling time is $10 \mathrm{~s}$.

The bar damage was generated by reducing the elastic modulus in the analysis. Two kinds of damage are considered in the paper, in which the elastic modulus was, respectively, reduced $40 \%$ and $80 \%$.

3.2. The Layout Scheme Measuring Point. After selecting excitation method, we should determine the number and

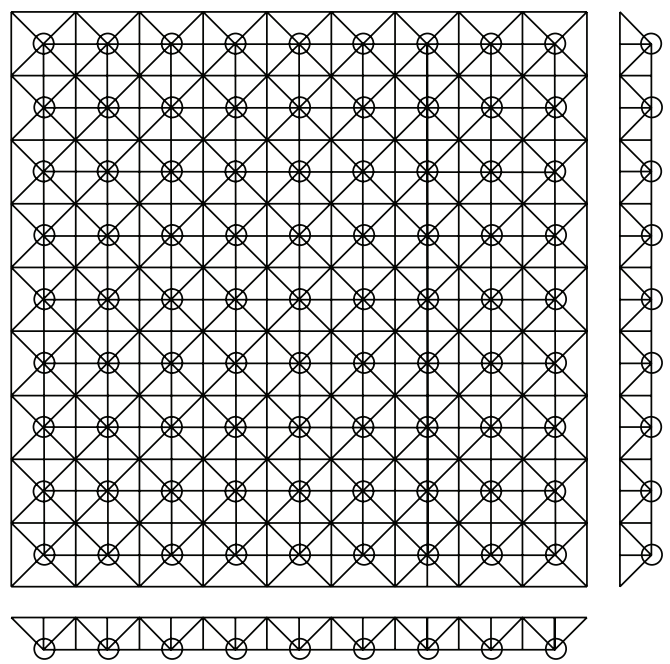

FIGURE 3: The layout of acceleration sensor.

the position of the measuring point. Because the upper chords are covered with roofing material, the acceleration response sensors were placed on bottom chord nodes. This was shown in Figure 3. Every sensor was fixed in $x$-axis direction.

The cross correlation function was calculated by using the responses of two adjacent acceleration sensors. The peaks of cross correlation functions formed a matrix as shown in (9). $r_{1,1-2}$ means a cross correlation function peak obtained from the sensor 1 and the sensor 2 in the first row. Consider

$$
R=\left|\begin{array}{c}
r_{1,1-2}, r_{1,2-3}, \ldots, r_{1,8-9} \\
r_{2,1-2}, \ldots \\
\vdots \\
r_{9,1-2}, r_{9,2-3}, \ldots, r_{9,8-9}
\end{array}\right| .
$$

The environment pulsation is a stationary signal. Its spectrum is almost unchanged. For the same damage, the peak matrixes obtained at different time are highly similar. By placing measuring points in different zones at different time, we can also get the whole peak distribution. When the grid size is very large, and the number of sensor is limited, the layout scheme as shown in Figure 4 can be used. The accelerometers were placed on the bottom nodes, evenly placed first, and then subregionally placed in the damage zone.

3.3. Damage Location. The damaged positions can be acquired through the abrupt changes of the peak matrix. Figure 5 shows the peak distribution of an intact grid. Figure 6 shows the peak distribution of a damaged grid. If the areas where the peak changes occur can be seen, the damage positions can be found.

In order to find the peak abrupt changes easily, the peak matrix could be processed as follows. After the matrix of a damaged grid subtracted the matrix of an intact grid, an abrupt change matrix was obtained as shown in Figure 7 . We can more easily identify the damage positions from Figure 7. 


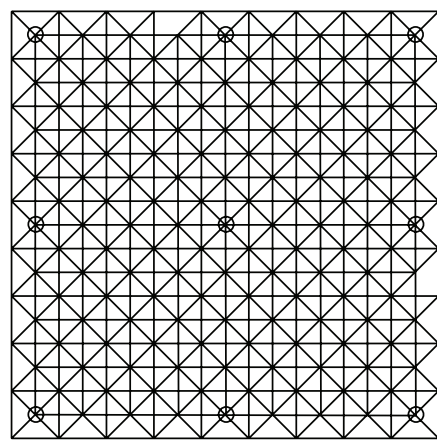

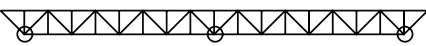


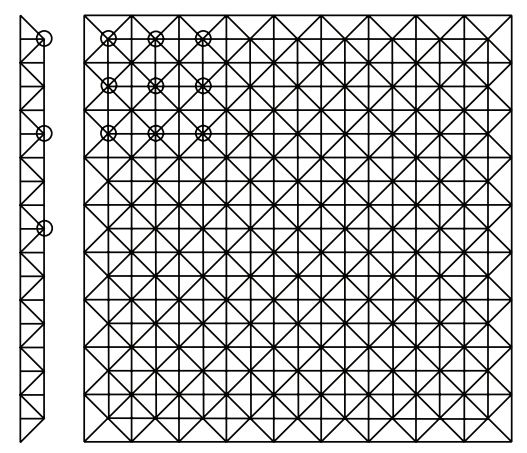

QS

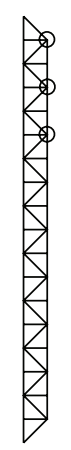

Figure 4: The subregional layout of acceleration sensor.

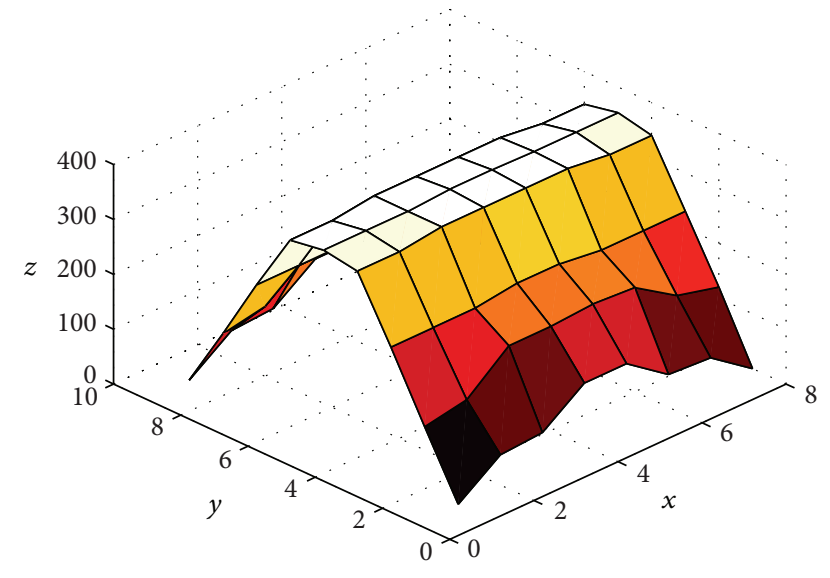

FIGURE 5: The peak distribution of an intact grid.

If the sensors were fixed in every bottom node along $y$ direction, we could obtain another matrix shown as (10). $r_{1-2,1}$ means a cross correlation function peak obtained from the sensor 1 and the sensor 2 in the first column. Consider

$$
R^{\prime}=\left|\begin{array}{c}
r_{1-2,1}, r_{1-2,2}, \ldots, r_{1-2,9} \\
r_{2-3,1}, \ldots \\
\vdots \\
r_{8-9,1}, r_{8-9,2}, \ldots, r_{8-9,9}
\end{array}\right| \text {. }
$$

Combining the distribution maps of abrupt changes derived from two directions, we can determine which quarter of the basic unit (as shown in Figure 8) involves damaged bars. Considering the case of node failure, each quarter has five damage modes: (1) the bottom chord along $x$ direction was damaged or off node; (2) the bottom chord along $y$ direction was damaged or off node; (3) the diagonal chord was damaged or off node; (4) the upper chord along $x$ direction was damaged or off node; (5) the upper chord along $y$ direction was damaged or off node.

3.4. Wavelet Packet Decomposition. The acceleration responses in damaged basic units were decomposed into 8

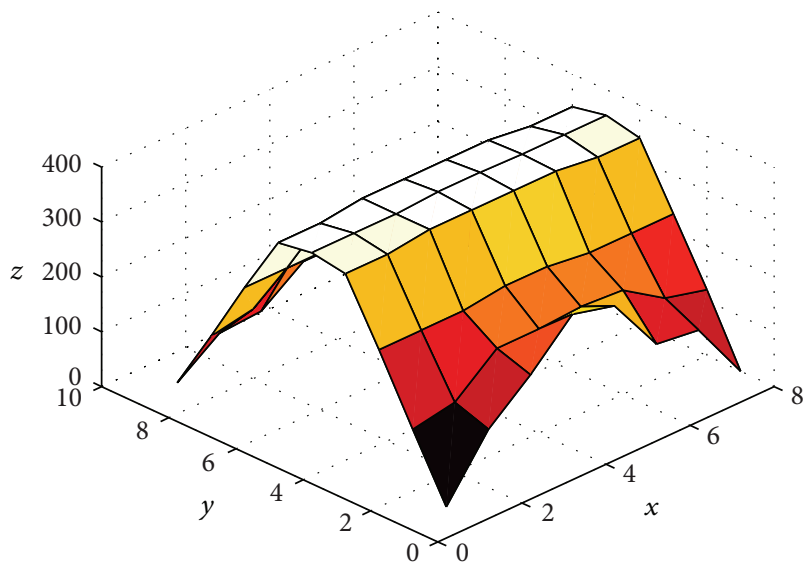

FIGURE 6: The peak distribution of a damaged grid.

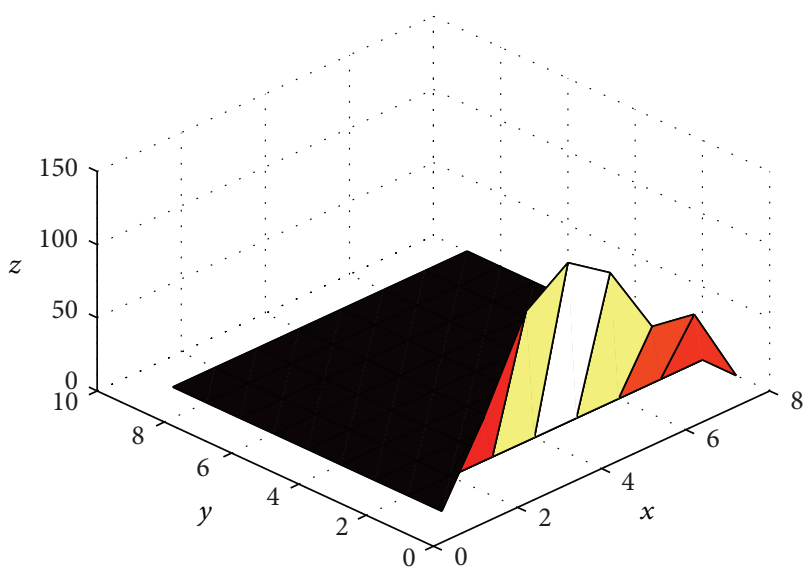

FIGURE 7: The abrupt change distribution of a damaged grid.

frequency components from low to high. After calculating the energy of every band, an 8-dimensional feature vector can be acquired and should be normalized, as shown in

$$
S=\left[E_{0}, E_{1}, E_{2}, \ldots, E_{7}\right]
$$


TABLE 1: Test accuracy.

\begin{tabular}{lccccc}
\hline $\begin{array}{l}\text { Damage } \\
\text { mode }\end{array}$ & $\begin{array}{c}\text { The bottom bar } \\
\text { element along } \\
x \text {-axis }\end{array}$ & $\begin{array}{c}\text { The bottom bar } \\
\text { element along } \\
y \text {-axis }\end{array}$ & $\begin{array}{c}\text { The diagonal } \\
\text { bar element }\end{array}$ & $\begin{array}{c}\text { The bottom bar } \\
\text { element along } \\
x \text {-axis }\end{array}$ & $\begin{array}{c}\text { The bottom bar } \\
\text { element along } \\
y \text {-axis }\end{array}$ \\
\hline Accuracy & $100 \%$ & $70 \%$ & $90 \%$ & $50 \%$ & $50 \%$ \\
\hline
\end{tabular}

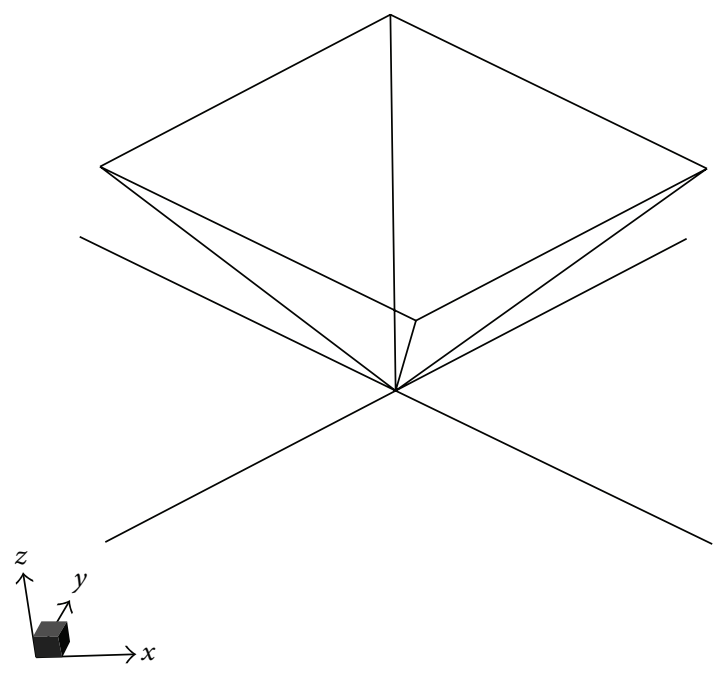

FIgURE 8: The grid structure module.

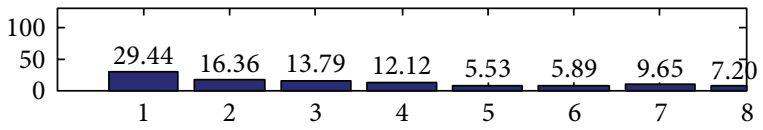

FIGURE 9: The energy distribution of intact base unit.

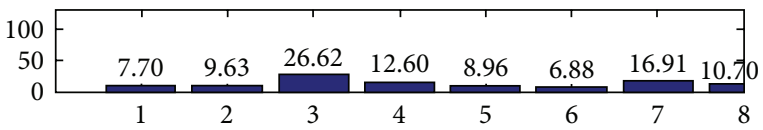

FIGURE 10: The energy distribution of bottom damaged chord in the $x$ direction.

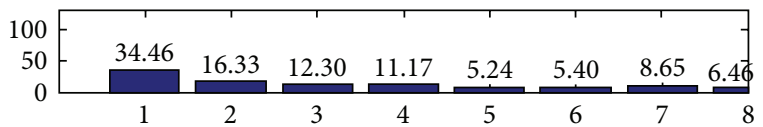

FIGURE 11: The energy distribution of bottom damaged chord in the $y$ direction.

For each basic unit, different damaged bars will produce different 8-dimensional feature vectors. The energy distribution of the intact mode is shown in Figure 9. The energy distributions of the 5 damage modes are shown in Figures 1014. A training sample of SVM was composed of the feature vector and the corresponding damage mode, while a test sample includes the feature vector only.

We collected 100 acceleration response signals as samples, which include five damage modes as samples. Every damage mode has 20 samples. Ten samples in each damage mode are

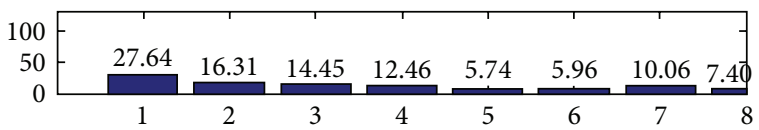

FIGURE 12: The energy distribution of damaged chord in the diagonal direction.

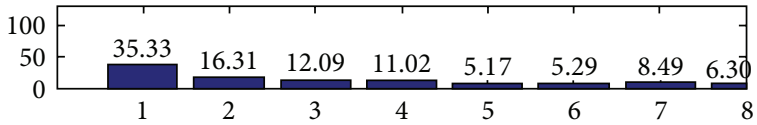

FIGURE 13: The energy distribution of upper damaged chord in the $x$ direction.

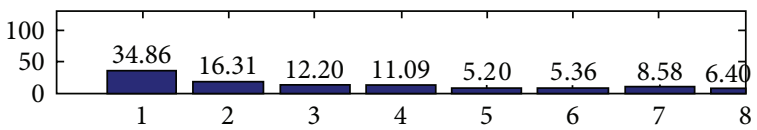

FIGURE 14: The energy distribution of upper damaged chord in the $y$ direction.

randomly selected as the training set. In the SVM, the kernel function is Radial Basis Function. The optimal $C$ and gamma were obtained by 5 -fold cross validation. The remaining 50 samples were used as test set. The accuracy of the test is shown in Table 1. As seen from the above results, the accuracy of the bottom bars is higher than the accuracy of the upper bars. The sensors are placed in the lower nodes; therefore, they are more sensitive to the damage of lower bars.

\section{Conclusion}

This paper established a damage location method which integrated a cross correlation function, wavelet packet decomposition, and support vector machine. The method was applied to a grid structure. The results show that the damaged bar elements in grid structures can be recognized accurately at controllable computation cost, which means less finite element calculation and less measuring points.

\section{Conflict of Interests}

The authors declare that there is no conflict of interests regarding the publication of this paper.

\section{References}

[1] D. J. Pines and A. E. Aktan, "Status of structural health monitoring of long-span bridges in the United States," Progress 
in Structural Engineering and Materials, vol. 4, no. 4, pp. 372$380,2002$.

[2] H. S. Sohn, C. R. Farrar, F. M. Hemez et al., A Review of Structural Health Monitoring Literature 1996-2001, Los Alamos National Laboratory, San Francisco, Calif, USA, 2003.

[3] S. Chen and G. Li, "Artificial neural networks in structural damage identification," Vibration, Test and Damage, vol. 21, no. 2, pp. 116-124, 2001.

[4] M. Kaouk, D. C. Zimmerman, and T. W. Simmermacher, "Assessment of damage affecting all structural properties using experimental modal parameters," Journal of Vibration and Acoustics, vol. 122, no. 4, pp. 456-463, 2000.

[5] S.-L. J. Hu, H. Li, and S. Wang, "Cross-model cross-mode method for model updating," Mechanical Systems and Signal Processing, vol. 21, no. 4, pp. 1690-1703, 2007.

[6] A. Dixit and S. Hanagud, "Damage localization by isolating the part of the response due to the damage only," Journal of Applied Mechanics, vol. 80, no. 1, Article ID 011015, 8 pages, 2012.

[7] M. R. Hernandez-Garcia, S. F. Masri, R. Ghanem, E. Figueiredo, and C. R. Farrar, "An experimental investigation of change detection in uncertain chain-like systems," Journal of Sound and Vibration, vol. 329, no. 12, pp. 2395-2409, 2010.

[8] A. Alvandi and C. Cremona, "Assessment of vibration-based damage identification techniques," Journal of Sound and Vibration, vol. 292, no. 1-2, pp. 179-202, 2006.

[9] J.-G. Han, W.-X. Ren, and Z.-S. Sun, "Wavelet packet based damage identification of beam structures," International Journal of Solids and Structures, vol. 42, no. 26, pp. 6610-6627, 2005.

[10] E. Figueiredo, M. D. Todd, C. R. Farrar, and E. Flynn, "Autoregressive modeling with state-space embedding vectors for damage detection under operational variability," International Journal of Engineering Science, vol. 48, no. 10, pp. 822-834, 2010.

[11] L. Liu and G. Meng, "Vector regression algorithm applied research in the beam structural damage diagnosis support," Shock and Vibration, vol. 25, no. 3, pp. 99-100, 2006.

[12] Y. Zhefu, H. Linsheng, and Z. Lei, "Grid structure damage location base on cross-correlation function," in Proceedings of the 4th International Conference on Civil Engineering, Architecture and Building Materials, pp. 1083-1086, May 2014.

[13] R. J. Allemang and D. L. Brown, "A correlation coefficient for modal vector analysis," in Proceedings of the 1st International Modal Analysis Conference \& Exhibit (IMAC '82), pp. 110-116, November 1982.

[14] L. Jiayan, Y. Qianfeng, L. Ying et al., "Experimental analysis of structural damage identification based on random vibration response of the cross-correlation function," Vibration and Shock, vol. 30, no. 8, pp. 221-224, 236, 2011.

[15] V. N. Vapnik, The Nature of Statistical Learning Theory, Springer, New York, NY, USA, 1995. 

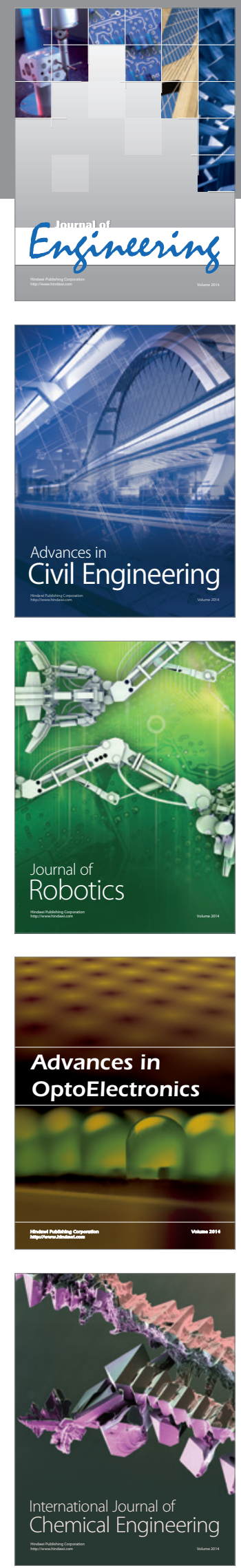

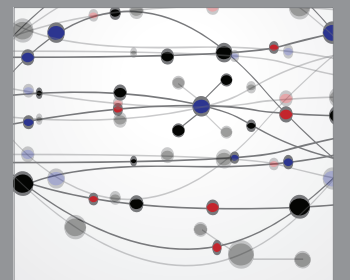

The Scientific World Journal
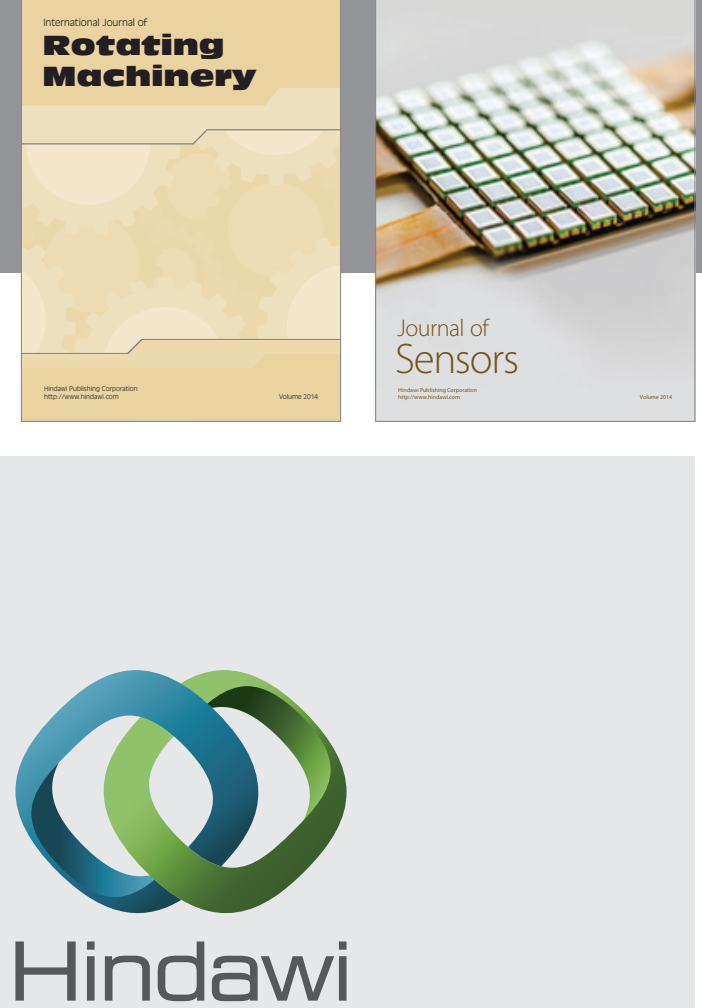

Submit your manuscripts at http://www.hindawi.com
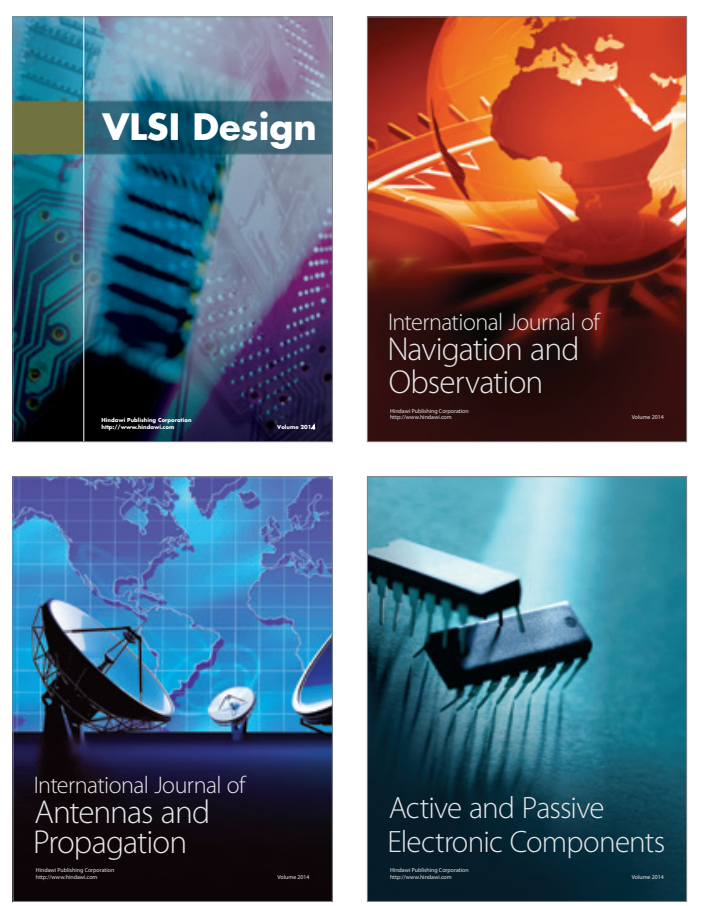
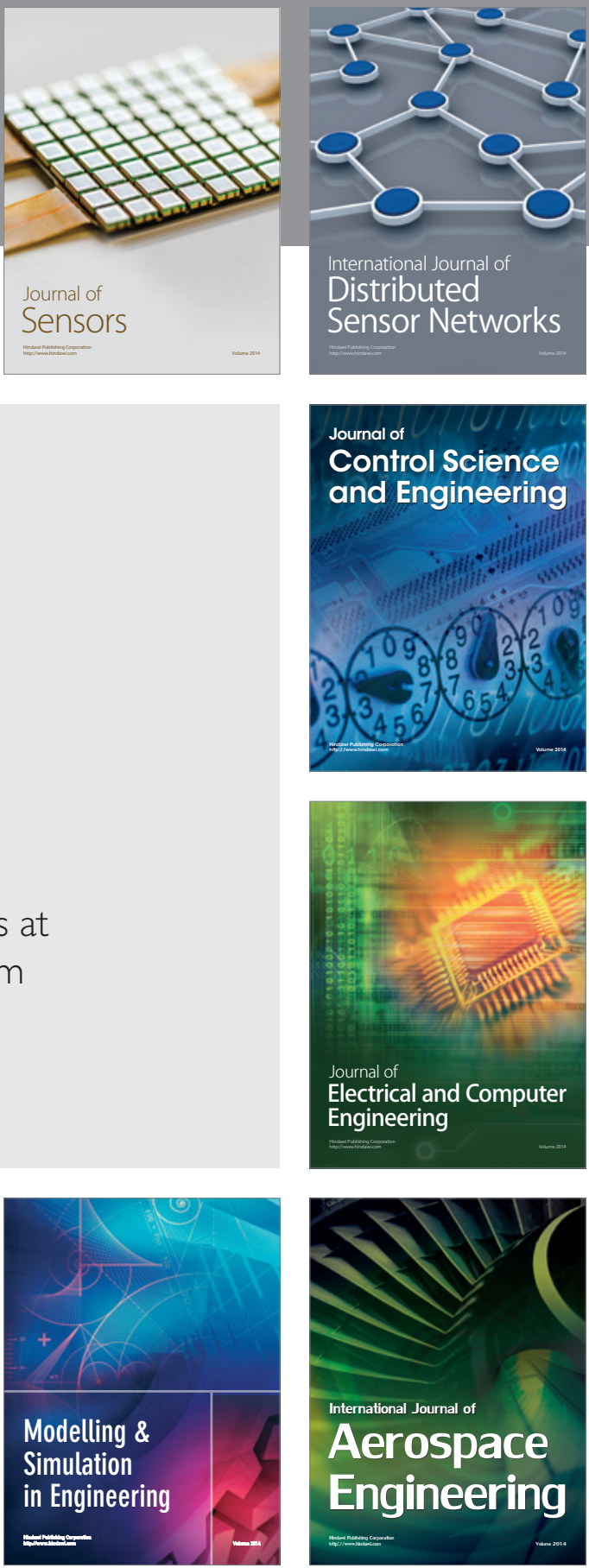

Journal of

Control Science

and Engineering
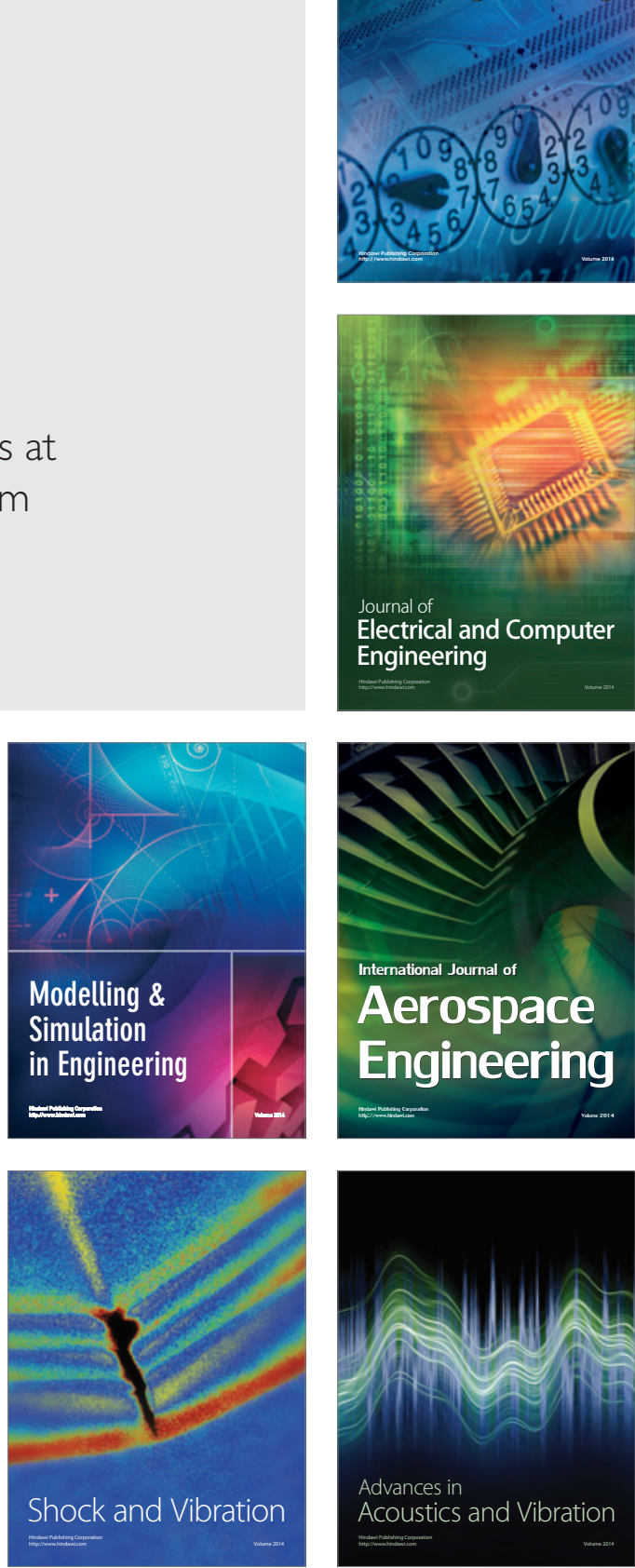